\title{
EC Vacuum Vessel Finite Element Analysis
}

Engineering Note \# 3740.224-EN-196

Issued: February 4, 1992

Originator: Dave Rudland/Rick Luther

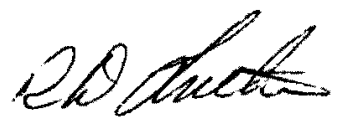




\title{
EC Vacuum Vessel Finite Element Analysis
}

\author{
Dave Rudland/Rick Luther
}

This Note contains a summary of the results of the finite element analysis of the EC Cryostat vacuum vessel performed by Dave Rudland in 1987. The results are used in the structural evaluation of the EC cryostats presented in Engineering Note 194. It should also be noted that the adequacy of the design of the vacuum vessels was reviewed and verified by the Battelle Memorial Institute. Battelle used a shell of revolution program to essentially duplicate the FEA analysis with similar results.

It should be noted that no plots of the finite element mesh were retained from the analysis, and these can not be easily reproduced due to a change in the version of the ANSYS computer program shortly after the analysis was completed. 
Uocum Uozol C+ il ring Buckling

Outer, Ring

$F_{1}=$ Force due to hoop stress on Vessel

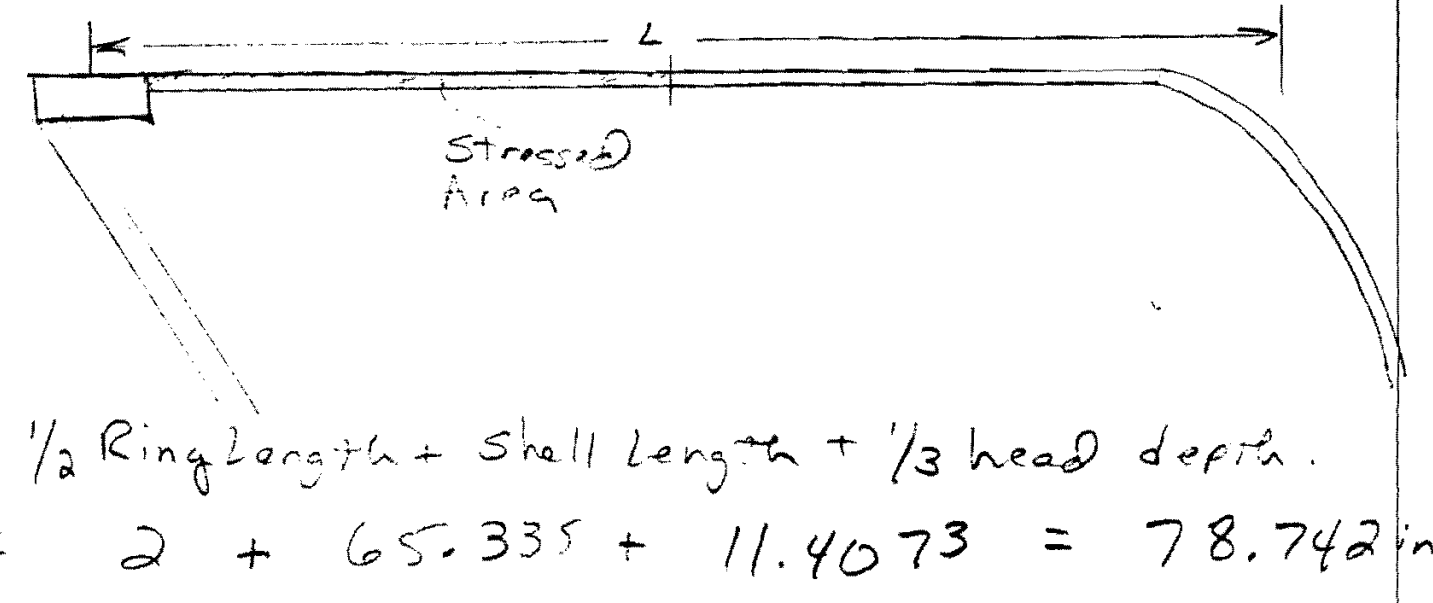

$$
\begin{aligned}
& F=\sigma \times A \quad \sigma=\frac{P E}{t}=\frac{15 \mathrm{psi}(102)}{-62}=2467.7419 \mathrm{pl} i \\
& A=(4 / 2-2) t=\left(\frac{78.742}{2}-2\right)(.62)=73.17 \mathrm{mi} \\
& F_{1}=2.67 .74 \mathrm{ipsi}+23.17=57177.631 \mathrm{bs}
\end{aligned}
$$

$F_{a}=$ Force due to hop stress on Ring And tar. Average Membrane hoof ind.

$$
i=1154 \times 65 \%
$$

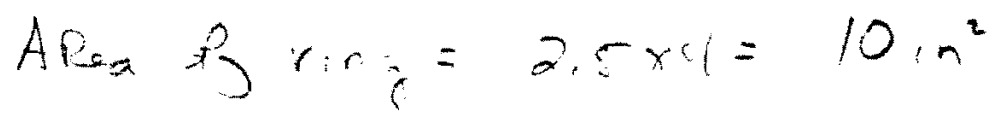

$$
F_{i}=115426.5 \mathrm{lbs}
$$

$F_{3}=$ Force on Window section $\frac{1.1 \sqrt{D_{0} t}}{2}$ sing is $=408 " ; t=.625$

$$
D=\frac{1.1 \sqrt{D_{0} E}}{2}=.55 \sqrt{408(.625)}=8.783
$$




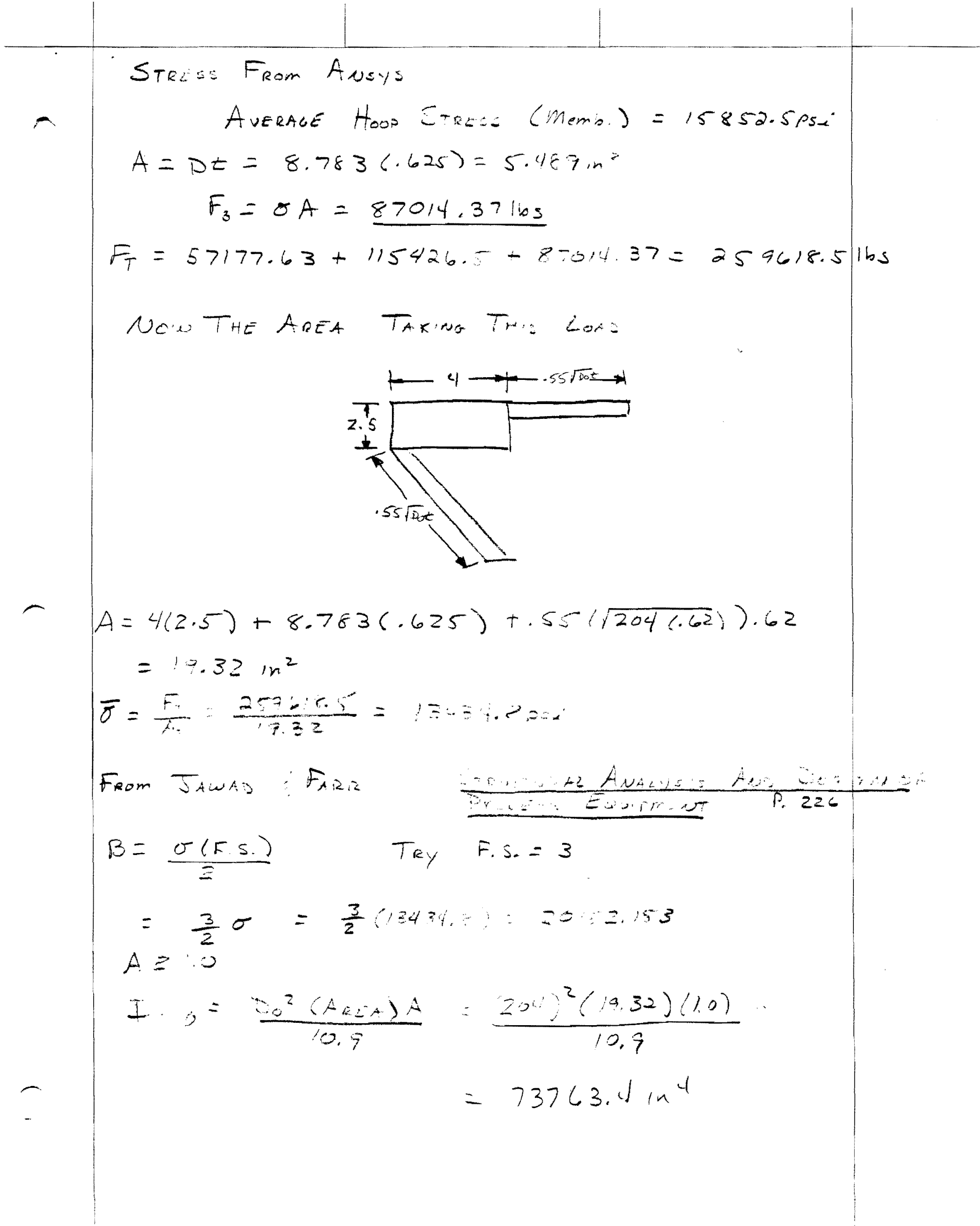




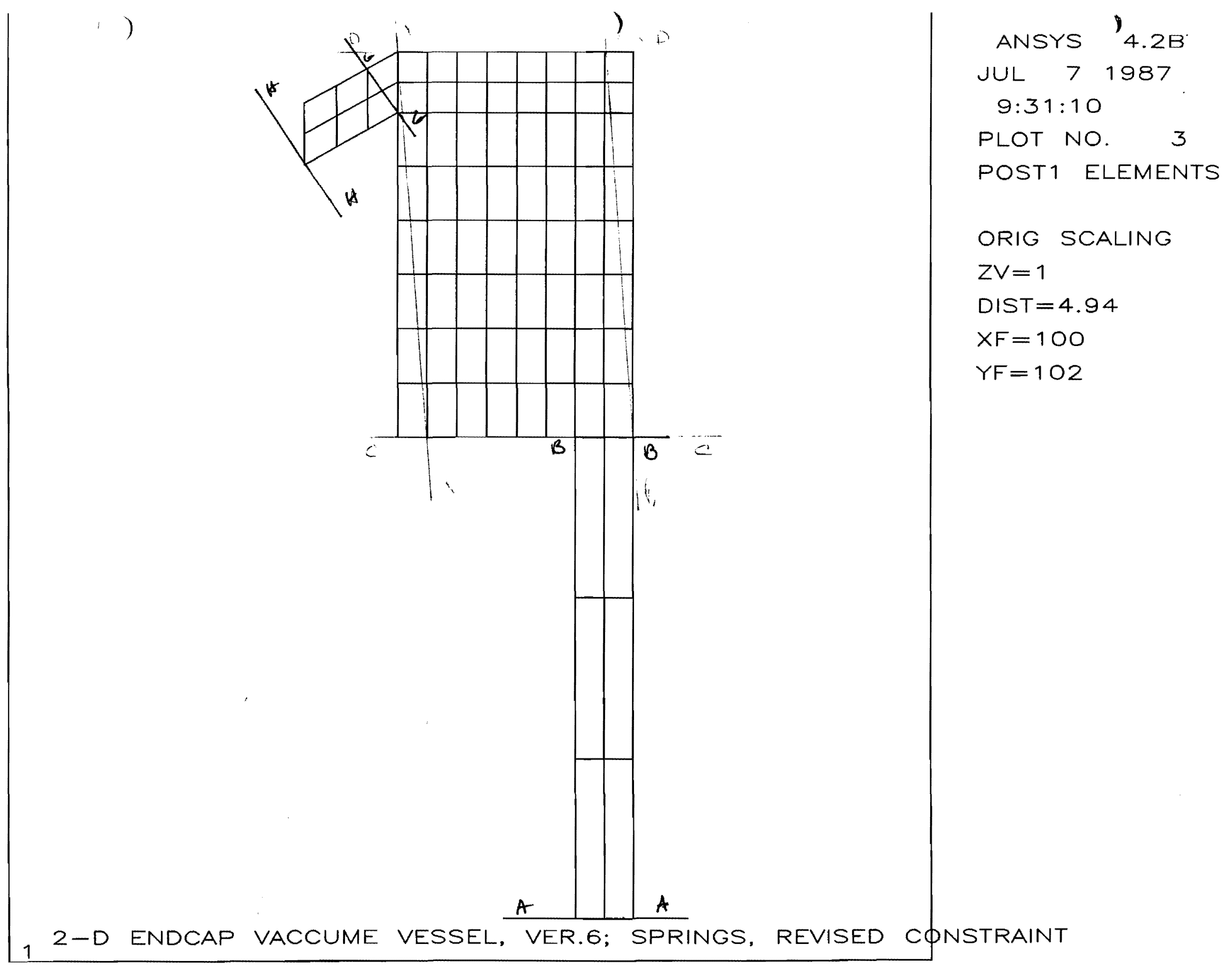




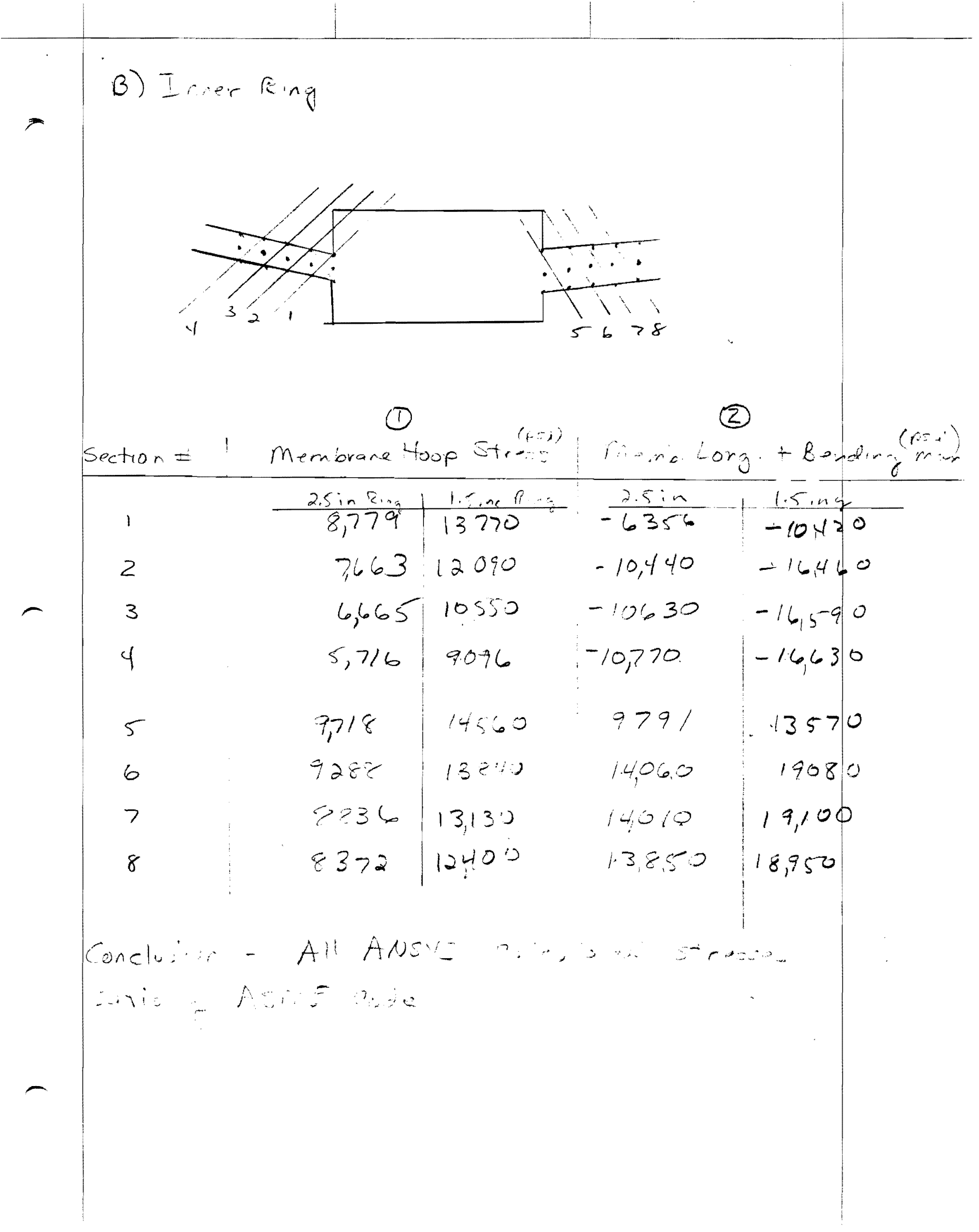




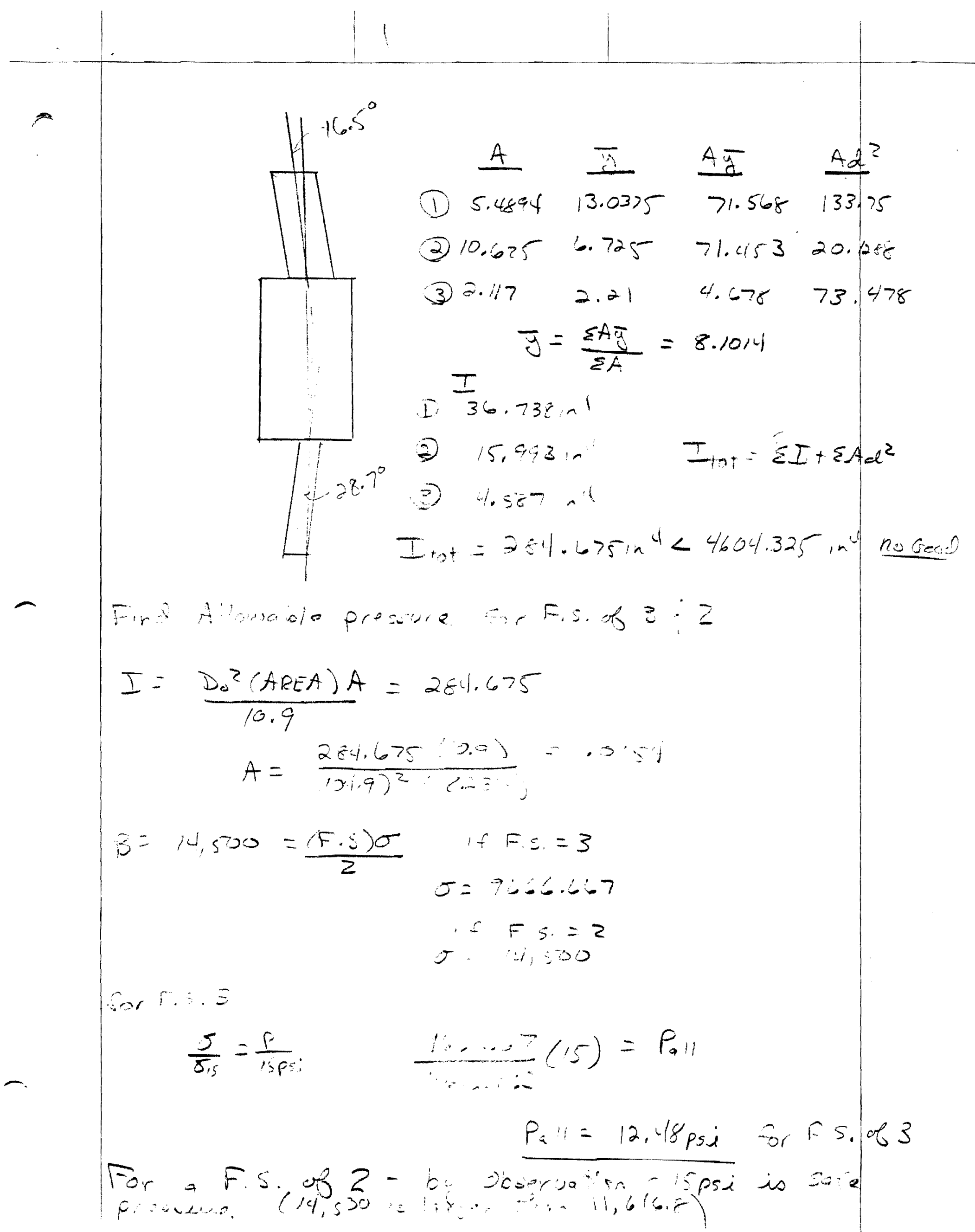




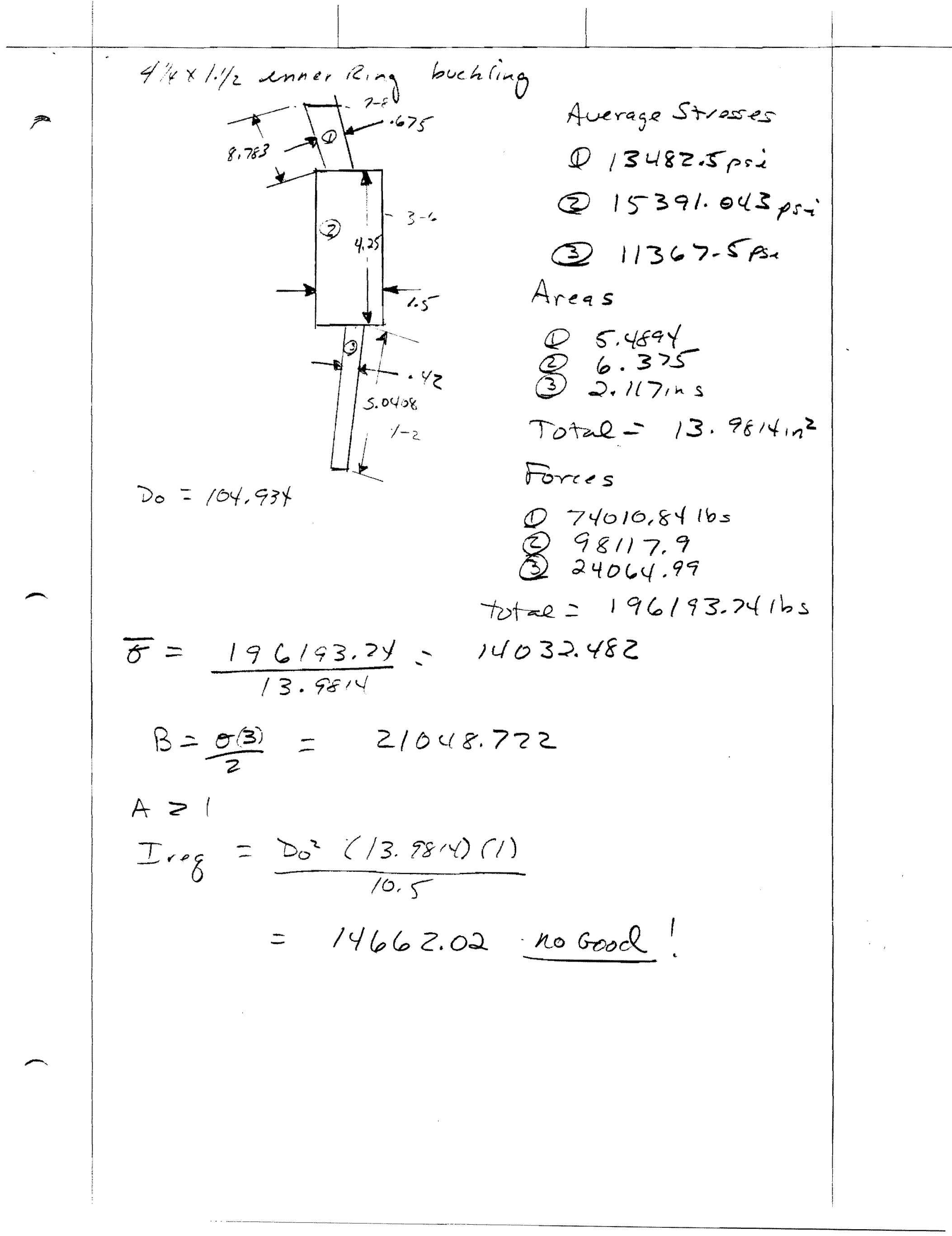


Calculate Actual I

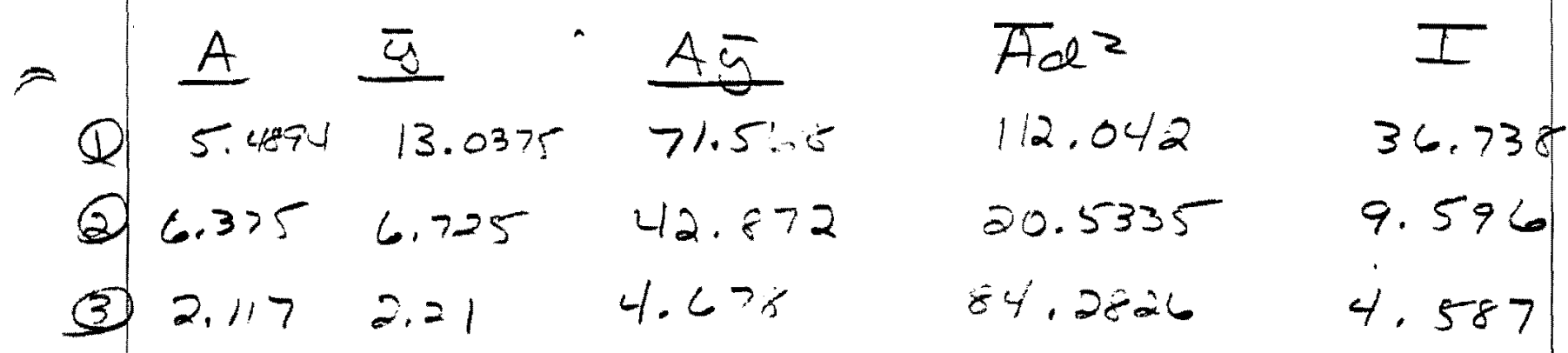

$$
\bar{y}=\frac{\sum A \bar{y}}{\sum A}=8,5197
$$

Itot $=E I+1 d \geqslant=267.7788 . \mathrm{n}^{\prime \prime}$

Find Allowable Pressure for F.S. of $3: 2$

$$
\begin{array}{r}
I=\frac{D_{0}^{2} \text { (AREA)A }}{10.9}=267.7788 \\
A=\frac{267.7788(10.9)}{(04.9)^{2}(13.9814}=.01897 \\
B=15,000=\frac{\text { Es (O) } \quad, f f . s=3}{2} \quad \sigma=10000 \\
1 f f .5=2 \\
\sigma=15,000
\end{array}
$$

for F.E $=3$

$$
\begin{aligned}
\frac{\sigma}{\sigma, 5}=\frac{p}{15} \quad P & =\frac{10,000}{14032.48}(15) \\
& =10.69 p \text { si for A.s. }=3
\end{aligned}
$$

For F.S. $=2$ - bug otserwation 15pai in

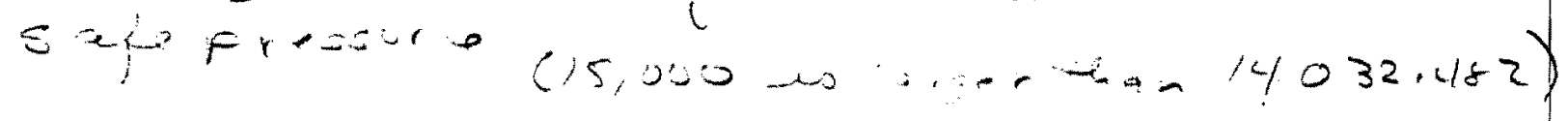




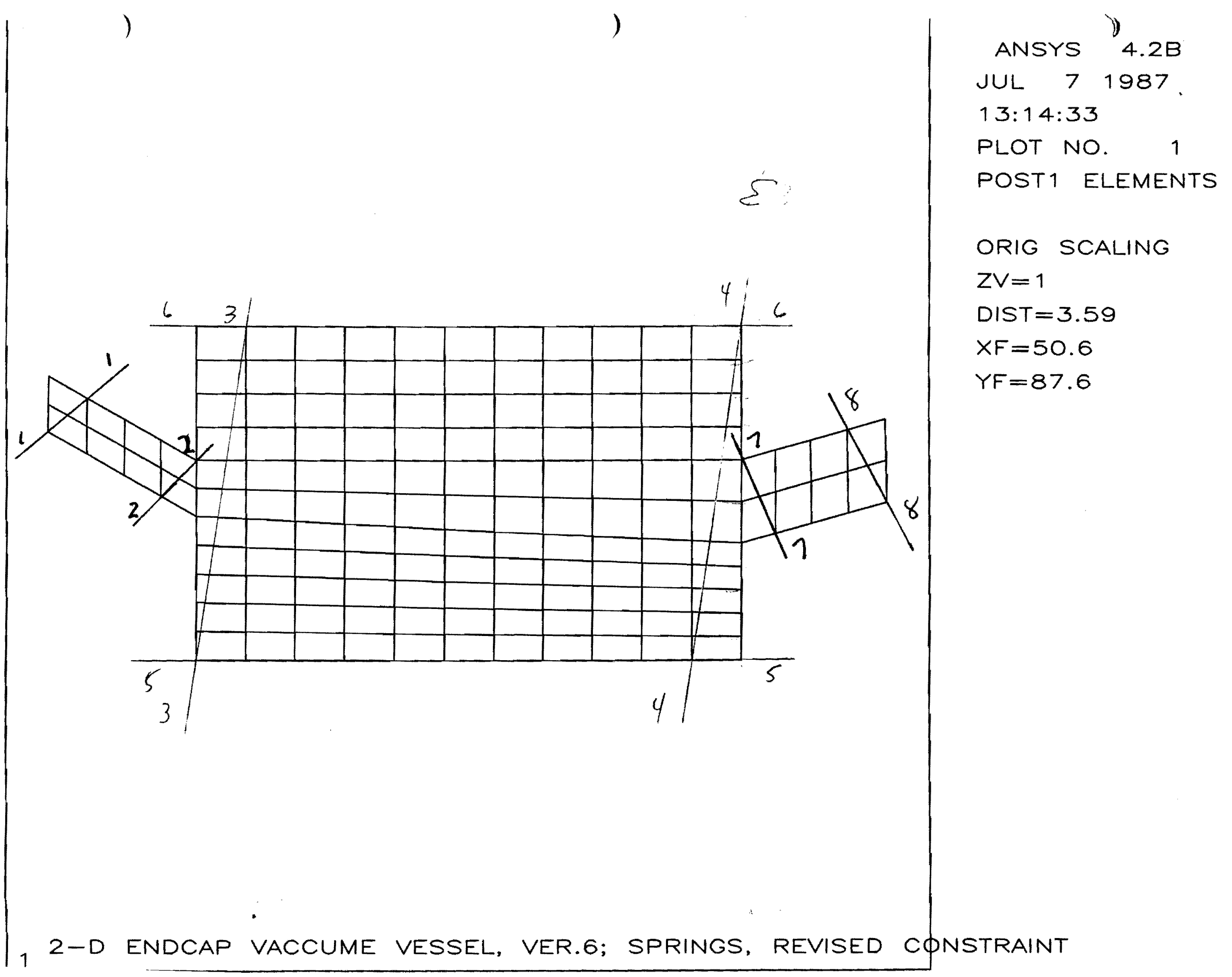

